the chemist for examination, but the rosult of examination for iron is negative; there appears to be very little in them.

The question of the influence of roller milling in the causation of appendicitis is one which has interested me for some years. Facts are accumulating to show that it is a serious factor, but this is not the occasion on which to consider it. There is, however, very little doubt that in addition to the small sharp particles which result from the disintegration of the fluting of the rollers, and which sometimes cause localised ulceration of the mucous membrane, there is a considerable amount of "iron dust," which is given out in such quantities that in certain individuals it can produce an actual blackening of the mucous membrane over extensive portions of the large bowel. This pigmentation is more extensive than could be produced by blood extravasations, and is more uniformly diffused.

Harley-street, W.

\section{THE THEORY OF SALICYLATE THERAPY IN RHEUMATIC INFECTION.}

By REGINALD MILLER, M.D. LOND., M.R.C.P. LCND., PHYSICIAN TO OUT-PATIENTS AND LECTURER IY PHARMACOLOGY, ST. MARY'S HOSPITAL; PHYSICIAN, PADDINGTON GREEN CHILDREN'S HOSPITAL.

ANY theory formulated to explain the action of the salicylate group of drugs in acute rheumatism has one great difficulty to overcome. It is briefly this. In some rheumatic manifestations, notably arthritis, salicylate is so successful in its action as to suggest strongly that the drug has an inhibitory power over the activity of the infecting organism; while in others, particularly chorea and carditis, such a power is by no means obvious. It is this so-called "selective" action of salicylate on the various manifestations of rheumatism which is responsible for the variety of views held upon its method of action.

In a recent paper ${ }^{1} I$ studied some of the practical objections which have been urged against the use of salicylate as a "specific" remedy in acute rheumatism, but purposely refrained from dis cussing the truth of the theory on which it is based. Here it is intended to support this "specific" theory, using the word "specific" as implying that the drug has the power of diminishing the activity of the rheumatic infection; the argument adopted being that this theory becomes acceptable when, but only when, the method by which the rheumatic infection produces its various results is properly understood.

\section{Modus Operandi of Rheumatic Infection.}

The modern view of rheumatism is that it is due to an infection by a particular type of micro-organism. This can be supported on strong clinical grounds as well as by the results of bacteriological research which are still unaccepted by some authorities. How this infecting organism produces its results has been made clear by the histo-pathological researches of Coombs, ${ }^{2}$ supplementing earlier work of Poynton and Paine and others. He finds evidence that rheumatic infection is a bacteriæmia which results in three sets of tissue changes: 1 . Interstitial inflammatory changes of a peculiar and characteristic type in the organs attacked by the infection. 2. Parenchymatous toxic changes from toxins produced from foci of infection, either local or distant. These changes reach their maximum significance where important tissues, such as the cerebral cortex or myocardium, are involved. 3. Sclerotic changes connected with healing processes.

Molus Operandi of Salicylate Treatment.

It is evident that a drug possessed of the power of diminishing the activity of the rheumatic infection could not control these three sets of tissue changes with equal success. 1. The inflam. matory processes should be well under the control of an antirheumatic drug. Assuming that the foci of infection were open to the drug circulating, its only limitations would be concerned with the virulence of the infection compared with the amount of the drug which could operate. 2. The toxic parenchymatous changes, on the other hand, could not be under the direct control of the drug. All that such a remedy could do here would be to cut short the supply of toxin at its sources, and thus prevent further damage. 3. The sclerotic changes obviously cannot be influenced by an antirheumatic drug, and need not be further discussed.

Clinically, then, salicylate, if possessed of a specific antirheumatic power, would control such rheumatic manifestations and symptoms as are dependent upon active inflammatory processes, but could only indirectly influence those dependent upon toxic parenchymatous changes. I must now review the chief rheumatic manifestations, attempt. ing to show that their reaction to salicylate conforms to this theory.

Arthritis.-The histological changes here are those of acute inflammation of the infected tissues within and around the joint. Toxic parenchymatous changes are of minimal importance here, as there are no "noble tissues" whose degeneration might produce symptoms. Under salicylate treatment there is a rapid subsidence of the local signs of inflammation together with a fall in general temperature. This last is of special importance, particularly when it is remembered that such a fall in temperature is not produced so markedly nor so constantly in any other infection, and therefore cannot be due merely to the slight general vasodilatation caused by salicylate. A fall in temperature, occurring in conjunction with local amelioration and general constitutional improvement, is generally held to be the best evidence of diminished bacterial activity, and it is certainly difficult to account for the success of salicylate in the treatment of rheumatic arthritis except by allowing a specific antirheumatic action to the drug. Another result confirms this view-namely, the way in which salicylate puts a stop to the development of arthritis in other joints which is so characteristic of the untreated infection.

There is one limitation to the successful action of salicylate on rheumatic arthritis - that concerned with large effusions. These may remain for a week or so before disappearing, even though the temperature has fallen and the other local signs have cleared up. The fluid obtained, however, from a joint at this stage will be found not only to be sterile but to show practically no cells. It shows, that is to say, no signs of bacterial activity, and it is evident that no antirheumatic drug could have an action upon such an effusion. Its absorption is necessarily and mainly a matter of time.

The action of salicylate on rheumatic arthritis shows well how the drug successfully controls such symptoms as depend upon bacterial activity, and 
fails to control such as persist after all signs of such activity have passed. The antirhoumatic action of salicylate is seen at its best in rheumatic arthritis because it is here that the symptoms due to inflammatory change are at a maximum, and those due to toxic parenchymatous change at a minimum.

Chorea.- In chorea we have histological evidence of two sets of changes in the central nervous system: (a) perivascular inflammatory changes from the presence of infecting organisms; and (b) toxic changes in the cells of the cerebral cortex. Before discussing the action of salicylate we must consider how far each of these sets of changes is responsible for symptoms in cases of chorea.

In certain acute febrile cases it is clear that we are dealing with an actual infection of the central nervous system. Poynton and Holmes ${ }^{3}$ have produced post-mortem evidence of this, which has been corroborated by Coombs. It is also confirmed in some non-fatal cases by the discovery of streptococci and cells in the cerebro-spinal fluid during the acute stage of the disease. On the other hand, it is probable that many slight cases of chorea are due to a rheumatic cerebral toxæmia rather than a cerebral infection. Of much more importance, however, is the recognition of the fact that the nervous symptoms of chorea may persist long after all rheumatic bacterial activity has ceased either in the brain or elsewhere, being dependent upon the damage already done by the infection to the cerebral cortex. That this is the case is shown by the normal (or subnormal) temperature and the normal cerebro-spinal fluid in many cases still showing the nervous symptoms of chorea.

No antirheumatic drug, therefore, can put an end to choreic movements. The most it can do is to diminish the activity of the infection, relieving such symptoms as are directly dependent upon such activity, and thus to limit the supply of toxin which damages the cerebral cells. These latter are then in a position to undergo repair, which when accomplished relieves the nervous symptoms. The fact, then, that salicylate cannot "cure chorea" in no way invalidates the theory that it has the power of diminishing the activity of the rheumatic infection. Indeed, the generally accepted view that salicylate is useful in chorea where signs of active infection (fever, pains, arthritis) are present is in accordance with this theory. When these have ceased salicylate, no matter in what large doses, can do no more to check movements, and at this stage treatment should aim at restoring the structure and function of the cerebral cells by rest, feeding, re-education, and such measures.

Carditis.-Here again we find two sets of tissue changes: inflammatory changes in the valves, pericardium, and interstitial tissue of the heart muscle, with toxic parenchymatous changes in the myocardium. Of these it is now generally recognised that the latter are productive of most of the ordinary cardiac symptoms, the inflammatory changes producing such symptoms as fever, friction, and possibly certain murmurs. The usefulness of salicylate, however strongly antirheumatic its action may be, will therefore be limited in carditis as in chorea. We cannot expect it to relieve the symptoms due to myocardial degeneration any more than we can expect it to remove the signs of mitral stenosis; on the other hand, we must expect evidence of its power to diminish the activity of the infection in the heart and the symptoms dependent upon it.
Such evidence will be forthcoming to anyone who has used salicylate to test this particular point. But very convincing cases are not numerous, because in children most cases of acute carditis show evidence of severe toxic myocardial degeneration, and in adults many are associated with a severe failure of myocardial power. Perhaps the most striking cases are seen in adults who, with a recent history of rheumatic arthritis, show ferer, a quickened pulse, and a mitral bruit without much cardiac dilatation. In such cases, which appear to be examples of acute carditis in which the inflam. mation is acute but the parenchymatous changes slight, salicylate successfully reduces the tempera. ture to normal and puts an end to the infective process. Similar results may sometimes be seen in first attacks of endo- or peri-carditis in children where there is no considerable dilatation of the heart. Even where the myocardium is severely degenerated the patient's temperature will often react to salicylate treatment if well tolerated, but this will not be associated with any immediate relief to the symptoms of cardiac dilatation.

This inability to cope with the myocardial degeneration and the symptoms thereof constitutes the first limitation to the practical use of salicylate in cardiac rheumatism. But there is a second, again associated with the state of the heart muscle. I have shown elsewhere ${ }^{1}$ that the amount of vomiting produced under salicylate treatment depends much more upon the state of the heart than upon the dose of the drug employed. Where the heart is only slightly affected vomiting is only troublesome in 4 per cent. of the cases, and now that the proper methods of administration are better understood practically should not occur. On the other hand, where the heart is much affected, particularly where it is severely dilated, troublesome vomiting occurs in about 30 per cent. of the cases, and in a large proportion of these is directly attributable to the drug, even though very small doses are employed.

Nodules. - These consist of macroscopic collections of inflammatory products, probably the results of bacterial emboli in end-arteries. We cannot tell how long a nodule may be expected to remain, so that we cannot tell if salicylate directly hastens their absorption. In investigating the theory of salicylate action the matter of rheumatic nodules does not assist us. All that one can say is that if salicylate can put an end to rheumatic activity it is probably doing all that is possible to hasten the disappearance of nodules. I have elsewhere ${ }^{1}$ discussed nodule formation as a rheumatic relapse under salicylate treatment.

Pyrexia.-As in other infections the temperature in rheumatism is a most important index to the activity of the infection. The fall in the temperature in the rheumatic arthritis of adults, coinciding as it does with marked local and general improvement, gives the strongest support to the specific claim of salicylate.

On the other hand, there is often seen, partioularly in cases of cardiac rheumatism, a "saw-like" temperature with about $2^{\circ}$ difference between the morning and evening readings, the latter reaching $101^{\circ}-2^{\circ} \mathrm{F}$. This is sometimes referred to as the fever of the "rheumatic state." but it denotes, I am convinced, some activity on the part of the infection. It is often stated that salicylate does not control this type of rheumatic pyrexia, but in my experience it is usually satisfactorily modified by 
an increase in the daily dose of salicylate to, say, $100-120 \mathrm{gr}$.

In more severe cardiac cases, where there is evidence of advanced myocardial degeneration, a different form of temperature is often seen. It is characterised by being very irregular, usually keeping below $100^{\circ}$. How far this is dependent upon bacterial activity it is difficult to determine; possibly in many cases it is due rather to the absorption of toxic material from degenerated myocardial cells. At all events, salicylate has seldom any beneficial action here even when used without causing symptoms of intolerance, which are so quickly aroused in cases of this type.

In practice I have come to lay stress upon the regularity or "saw-like" feature of the temperature. Where this is absent and the temperature is seldom above $100^{\circ}, I$ think severe cardiac cases are little benefited by salicylate, and may easily be harmed by it if nausea or romiting be induced. But where the temperature is persistently raised at night, even though the evening temperature is quite low, I prefer to continue with salicylate if possible, as I think that under these circumstances there is some smouldering activity of the infection present. My chief reasons for this conclusion are (a) that this type of temperature usually develops from a similar saw-like temperature of higher grade; (b) that relapses of pyrexia are frequent; and $(c)$ that the patient's improvement is seldom satisfactory until this type of temperature ceases.

In some instances rheumatic hyperpyrexia can be reduced by salicylate, but here it is evident that we are dealing with an extremely virulent infection and with usually but little time in which to attempt to control it.

Taken as a whole, therefore, and with due allowance for the difficulties connected with parenchymatous cell changes, the action of salicylate upon the pyrexia of the rheumatic infection is in accordance with the theory that the drug has the power of diminishing the activity of the infection.

Tonsillitis.-Acute rheumatic tonsillitis appears to react well to salicylate treatment. The chronic forms, in which the organisms are buried deeply in the glandular crypts, cannot be expected to be much under the control of any drug circulating in the blood stream. This is of importance, since histological evidence suggests that in human rheumatism there are repeated reinfections of the blood. If these come, as is commonly assumed, from the tonsils, it is evident that an antirheumatic drug, if it could not destroy the organisms at their source, could only do so after they had gained access to the blood stream. This perhaps explains some of the relapses that may occur in patients recently under salicylate treatment or while the drug is being given in small doses.

Other manifestations.-Acute rheumatic nephritis and bursitis, both probably due to local infection, react well in my experience to salicylate. Rheumatic anæmia, on the other hand, is a toxic change and can be only indirectly benefited by the drug. It is noteworthy that this state is not improved by the administration of iron until the activity of the rheumatic infection is at an end. True rheumatic rashes are so irregular in their appearance and disappearance that the effect of salicylate upon them is hard to judge. Erythema nodosum and the socalled peliosis rheumatica I do not regard as truly rheumatic. "Rheumatic iritis" is excluded from the rheumatic group by many authorities, with whom I am inclined to agree.

\section{Other Theories of Sulicylate Action.}

The "specific" theory of salicylate action in rheumatism which I have supported above is probably widely held, although possibly not always with a just appreciation of exactly what it entails. On the other hand, it is well known that some of those most interested in rheumatic problems are strongly opposed to this view, and it is therefore necessary to examine briefly opposing theories.

Antitoxic theory.-A. E. Garrod ${ }^{4}$ has suggested that salicylate possibly possesses the power of neutralising rheumatic toxin rather than of attacking the infecting agent. Thus he writes:-

Can it be that they [the salicylates] exercise an antitoxic rather than a bactericidal action ? It must be granted, as Professor Stockman has pointed out to me, such a conception finds no support in the established facts of pharmacology, and that no parallel example can be quoted of a neutralising effect of a simple chemical compound, administered as a drug, upon bacterial toxins ; but neither can any other instance be adduced of such selective influence of a drug upon certain of the manifestations of a disease of bacterial origin.

F. J. Poynton ${ }^{5}$ seems to favour a somewhat similar view, more elaborated. Thus :-

I believe the bacterial poisons are so subtle that they vary with the tissues in which the lesions occur, and I think it probable that salicylates may possibly be specific to some of them and not to others.

In addition to the difficulty already quoted by Garrod, an important argument against the truth of this antitoxic theory lies in the fact that it is diametrically opposed to the evidence afforded by the histo-pathology of rheumatism. As I have attempted to show, it is those symptoms which are dependent upon inflammatory processes that are well controlled by salicylate, while those due to toxic parenchymatous changes are comparatively unbenefited. It would therefore seem that a bactericidal rather than an antitoxic action would best explain the "selective influence" of the drug.

Partial-specific theory.-This viow, for which I cannot find a more satisfactory name than that given, is widely held. A couple of quotations will demonstrate the theory. A. F. Voelcker ${ }^{6}$ writes: "We are not justified in regarding the salicylates as a specific for any and every rheumatic affection." Again, A. Abrams" writes: "Salicylates have a specific action only on the articular manifestations."

We must here pause to remind ourselves that the term "specific" may be applied to a remedy in two senses. It may be employed as meaning merely "peculiarly efficacious." Used in this sense, although the above statements become defensible, they are of little theoretical interest since no explanation of the drug's action is attempted. Employed, however, in connexion with a remedy for an infective disease, the term "specific" is usually meant to express that the drug has a bactericidal power of some sort over the infecting agent. Used with this meaning any "partial specific" view becomes full of difficulty. For if the modern view of rheumatism as a definite infection attacking various organs of the body be correct, it is clearly illogical to presume a bactericidal action on the organisms attacking one part of the body and to deny it to those producing similar lesions elsewhere. The only explanation of such a selec. tive action would be that in some organs the foci of inflammation become "closed" (as in tuberculosis) and thus unapproachable to a circulating drug, and of such a process there is no histological evidence; indeed, $_{3}$ the inflammatory foci appear closely connected with dilated vessels. 
The only possible alternatives to the specific view would appear to be either the "antitoxic theory" already considered or the "non-specific theory" to be discussed immediately. Poynton, who admits the difficulty, is drawn to both alternatives, but apparently is not well satisfied with either. To quote the passage in full $^{5}$; -

I do not dispute that some rheumatic lesions, notably arthritic and muscular pains, are greatly relieved and the temperature is lowered by these drugs, but further than that I am not prepared to go. How can such a position as mine be justifiable? I believe the bacterial poisons are so subtle that they vary with the tissues in which the lesions occur, and I think it probable that the salicylates may possibly be specific to some of these poisons and not to others. I am even tempted to feel that their specific action upon rheumatic arthritis is perhaps overrated, for $I$ have seen other acute forms of arthritis relieved by them. It does not follow, I think, because acute rheumatic arthritis subsides rapidly that it is the salicylates which produce this subsidence though they may relieve the pain ......

Non-specific theory.-It might be logically held that salicylate has no power to control the activity of the rheumatic organism wherever displayed. Some are drawn to this view, although nowhere can I find it very definitely put forward. How far Poynton is prepared to go in this direction has already been shown. L. G. Guthrie ${ }^{8}$ may also be quoted :-

Salicylates relieve pain and lower temperature. I do not think they cure rheumatism and am sure they do not avert cardiac affection. ...... Drugs may palliate but do not cure either rheumatism or chorea.

Before such a view can be accepted some explanation of the reaction of rheumatic arthritis to salicylate must be forthcoming, particularly in connexion with the fall of temperature, which, when accompanied by local and general improvement, is usually accepted as strong evidence of a lessening activity on the part of an infection. The only alternative to allowing a specific action to salicylate here would seem to be to deny that the drug acts any more successfully upon rheumatic than on any other form of infective arthritis. And this would be against general experience ; for, although everyone has seen some lowering of the temperature under salicylate in other infective conditions, yet it is generally acknowledged that as regards its degree, constancy, and permanence the fall of temperature in rheumatic arthritis under the drug is peculiar.

Chemical Support of Specific Theory.

Space will not permit me to do more than refer to Stockman's important article on the Action of Salicylates in Rheumatic Affections, ${ }^{9}$ in which he shows that the antirheumatic power of salicylate and chemically allied drugs depends upon the relative positions of the carboxyl and hydroxyl groups. He writes:-

The action of salicylic acid in acute rheumatism is undoubtedly specific. It is of little or no therapentical value in other fekrile diseases, and it exerts no action on the healthy man from which one could infer its action in rheumatic fever. But besides clinical evidence the chemical evidence points conclusively in the same direction.

\section{Conclusion.}

That salicylate has the power of diminishing the activity of the rheumatic infecting agent may be taken as the working basis of salicylate therapy in rheumatism, and the drug should be administered with the sole view of controlling the bacterial activity. Of this the temperature chart is as accurate an index in rheumatic as in other infections (e.g., tuberculosis). Care must be taken to discriminate between symptoms directly due to active rheumatic inflammatory processes and those dependent upon toxic parenchymatous changes which may remain long after all bacterial activity is at an end.

Note on dosage.-The temperature chart is the guide to the dose of salicylate to be employed. Used solely with a view to controlling bacterial activity, the effective range of dosage for child or adult is usually from 60 to 120 grains per diem. Less than 1 drachm daily is rarely sufficient; more than 2 drachms need seldom be given. Much larger doses than these have been shown to be safe if properly administered, and if necessary to gain control may be used, but for the most part "massive" doses have been administered in cases of chorea in which there was no evidence of bacterial activity remaining.

Any dose greater than 100 grains daily should be given, as Dr. D. B. Lees teaches, subdivided into 10 doses in the 24 hours. Large infrequent doses should never be given, particularly in children. Constipation must be aroided. The sodium salicylate should be combined with an equal dose of sodium bicarbonate; I have not found the double quantity of soda necessary in doses of this grade. Vomiting in cases of severe cardiac dilatation may be difficult or impossible to prevent, but in other cases may be avoided for the most part. The more serious symptoms of salicylate poisoning (acid intoxication) should nowadays never be allowed to arise.

Bibliography.-1. Miller: Quarterly Journal of Medicine, 1913, vol. vi., p. 519. 2. Coombs : St. Mary's Hospital Gazette, 1913, vol. ii., p. 22 (summary of previously published results). 3. Poynton and Holmes : THE LANCET, 1906, vol, ii., p. 982. 4. Garrod: Ibid., 1911, vol.i., p. 1411. 5. Poynton: Brit. Med. Jour., 1912, vol. ii., p. 932. 6. Voelcker : Medical Society's Transactions, London, 1911, vol. xxxiv. 7. Abrams : Brit. Med. Jour., 1912, vol. it.. p. 932. 8. Guthrie : Proceedings of the Royal society of Medicine (Therapeut Harley-street, W

\section{DRY CUPPING IN LARYNGEAL AFFECTIONS.}

By W. STUART-LOWV, F.R.C.S. ENG.,

SURGEON TO THE CENTRAL LONDON THROAT, NOSE, AND EAR HOSPITAL.

For some time I have been using dry cupping of the external surface of the larynx and adjacent area of the neck with very beneficial effect in some laryngeal affections, but $I$ can find no record in laryngeal literature of its employment for this class of disease. That dry cupping should not have been used for the relief and amelioration of laryngeal trouble is the more remarkable since it is so readily and so easily applied, and the anatomy of the parts, particularly the blood-supply, lends itself advantageously to such treatment.

The best and most easily worked apparatus for regular and systematic dry cupping is a suction apparatus (tankless), an American instrument, the agents for which in this country are Messrs. Rouse, of Wigmore-street. There are two sizes of this machine, the No. 20, with three cylinders suitable for consulting-room work, and the smaller portable one, which I have found quite sufficient and most useful at the bedside. I have had cups made of various sizes and shapes for adaptation to the front and sides of the larynx, and have found it best to have the edges of the cups covered with rubber so that any inconvenience the patient might feel when the suction is strongest may be minimised. Some of these cups are half-moon shaped, others horse-shoe shaped. Suction can be applied effectively to one or both sides of the larynx simultaneously. There is an adjusting mechanism on the instrument by 